\title{
High-precision time-of-flight determination algorithm for ultrasonic flow measurement
}

\author{
Francesc Suñol, Diego A. Ochoa, and Jose E. Garcia
}

\begin{abstract}
Commercial time-of-flight (TOF) ultrasonic flowmeters are rapidly expanding in the general industry. Among the different techniques that can be applied to determine the TOF of ultrasonic waves, the cross-correlation method presents numerous advantages, such as robustness for weak signals and noise suppression. However, the selection of an appropriate reference wave is presumably a key element in the precise measurement of TOF. In the present paper, an algorithm to compute an accurate TOF is proposed. The form of the electric signal received by the transducer is obtained from an acoustically-forced underdamped oscillator model, and the analytical solution of the model is proposed as a reference wave. In order to validate the effectiveness of this procedure, an ultrasonic flowmeter system is designed and tested in a flowmeter calibration test rig. It is demonstrated that the use of the presented scheme overcome the average method limitations, and turns out to be a convenient solution in a wide range of conditions. Robust measurements of near-zero flow values are acquired, which allow the achievement of a high dynamic range. The error curve of the proposed system have been obtained, revealing that the absolute value of the relative errors are lower than $2 \%$ within all the spectrum of flow rates considered (from 0.2 to $150 \mathrm{~m}^{3} / \mathrm{h}$ ). Results demonstrate that the algorithm provides high-precision measurements within a wide dynamic range. The algorithm is portable and versatile: it can be adapted to different types of transducers without the need of additional measurements, allowing to adjust parameters on-the-fly for an optimal performance of the ultrasonic flowmeter system.
\end{abstract}

Index Terms-Cross correlation, time-of-flight, fluid flow measurement, ultrasonic, flowmeter.

\section{INTRODUCTION}

$\mathbf{T}$ HE need for accurate and reliable flow measurement is growing in modern industry production area. Proper measurement is essential in processes in which liquids and gases are produced or transported. The use of ultrasonic flowmeters (UFM) is rapidly expanding in industrial, energy and medical fields because of the significant operational and economic advantages that the UFM offer in contrast to conventional meters [1-3]. Numerous methods of flow measurement using ultrasound have been developed, such as the time-of-flight (TOF, also called transit-time), doppler effect, or vortex and surface wave modulation. TOF UFM are gaining a lot of importance in the world of hydraulic metrology because they allow non-intrusive flow measurement (leading to low pressure drop), requiring very low maintenance (due to absence of moving parts) while offering high measuring precision in a wide dynamic range $[4,5]$. In virtue of these advantages,

F. Suñol. D. A. Ochoa and J. E. Garcia are with the Department of Physics, Universitat Politècnica de Catalunya-BarcelonaTech, Barcelona, Spain (email: francesc.sunol@upc.edu).
TOF UFM has become today's most popular technology of ultrasonic flow measurement $[6,7]$.

The operation of TOF UFM is based on a well-established principle: when an ultrasonic pulse propagates through the flowing medium, its propagation time will vary with the flow velocity of the medium. Two equal transducers $a$ and $b$ are located on each side of a pipe of diameter $D$, with a separation between them by a distance $L$ along the pipe axis, thereby forming an angle $\alpha=\arctan (D / L)$ with the flow direction (Fig. 1). Within this configuration, the TOF of the downstream signal $t_{a b}$ and of the upstream signal $t_{b a}$ can be expressed as [8]

$$
t_{a b}=\int_{0}^{H} \frac{d h}{c+v(h) \cos \alpha},
$$

and

$$
t_{b a}=\int_{0}^{H} \frac{d h}{c-v(h) \cos \alpha},
$$

respectively, being $H$ the acoustic path length, $c$ the velocity of the ultrasound wave, and $v(h)$ the axial flow velocity measured at point $h$ along the acoustic path. Integrating (1) and (2) and assuming that $v(h)$ is independent of $z$ (pipe axis direction), an expression for the mean flow velocity can be obtained in terms of TOF and geometrical parameters

$$
\langle v\rangle=\frac{D}{\sin (2 \alpha)} \frac{\Delta t}{\Pi t},
$$

where $\Delta t=t_{b a}-t_{a b}$ is the TOF difference between the upstream and downstream paths, and $\Pi t=t_{b a} t_{a b}$ is the product between TOFs. If the sound speed $c$ is known in advance, the mean flow velocity can be computed using the TOF difference by $\langle v\rangle=c^{2} \Delta t /(2 L)$ [9]. However, to measure accurately the sound speed is a truly challenging task, since it can be affected by temperature, chemical composition, presence of impurities, etc. An alternative strategy to this approach, is to measure the TOF of both upstream and downstream signals and compute the flow rate directly as the product of the mean flow velocity and the pipe section area, by

$$
Q=\frac{\pi D^{3}}{4 \sin (2 \alpha)} \frac{\Delta t}{\Pi t} .
$$

An accurate value of the sound speed can be then obtained by

$$
c=\frac{2 D}{\sin \alpha} \frac{1}{\Sigma t}
$$

where $\Sigma t$ is the sum of the upstream and downstream TOFs.

It can be noted that this principle of flow measurement operation allows bi-directional flow measurement. In practice, the flow velocity profile is not uniform at all points in the 


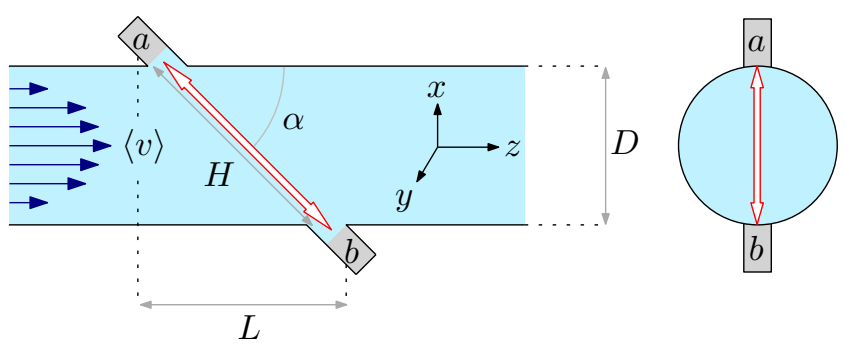

Fig. 1. Schematic illustration for the time-of-flight measurement principle. The acoustic path $H$ between two transducers $a$ and $b$ forms an angle $\alpha$ with the mean velocity $\langle v\rangle$. Transducers are separated a distance $L$ along a pipe with a diameter $D$.

pipe [10-12]. For this reason, some UFM models use multiple transducers, setting the acoustic paths off-centred in the cross section area of the pipe. In addition, a precise determination of the transducers exact location is a challenging task. These setbacks can be absorbed by introducing a calibration factor $K$, which require appropriate adjustment to allow for the combined effects of the transducer placement and the average flow rate in the pipe cross section for laminar, transitional, or turbulent profiles that can be either steady or unsteady [13$15]$. With this, the actual flow rate is measured as

$$
Q=K \frac{\pi D^{3}}{4 \sin (2 \alpha)} \frac{\Delta t}{\Pi t} .
$$

Under reciprocal operation, electric signals measured at the receiver transducers are equal and the TOF difference can be computed by performing a correlation on them. However, when reciprocal operation can not be achieved, signal waveforms with different magnitudes, envelopes, and even frequencies will be received on both sides, causing an error knwon as the "zero flow error". Bo et al. [16], used forced oscillations to reduce the zero-flow error for non-reciprocal ultrasonic flowmeters, by measuring the TOF difference as a phase difference between upstream and downstream signals [17]. Nonetheless, wihtin the proposed method signals are cross-correlated to a pre-computed reference signal. The parameters of the reference signal can be adjusted so not only the TOF difference is measured, but also the complete value of the TOF is obtained in non-reciprocal conditions. If the upstream and downstream values of TOF are known, an accurate value of the flow rate can be computed by means of (6). It can be seen from (6) that the accurate measurement of TOF is critical to the precision of UFM. Consequently, many technologies have been developed to measure TOF, among which are the zero-crossing technique and the cross-correlation method [7, 18]. Based on the zero-crossing technique, current methods using Time-to-Digital Converter (TDC) can provide a precision of several tenths of picoseconds. Texas Instruments reported [19] an 80 ps peak-to-peak jitter and TOF difference offset drift of 25 ps measured at static (no-flow) conditions. Recently, Hamouda et al. [20] proposed a technique with a claimed precision of $10 \mathrm{ps}$ peak-to-peak jitter and zero offset drift at static conditions. However, most accurate strategies entail a trade-off between measurement precision and system complexity.
The zero-crossing technique has a low computational cost compared to the cross-correlation method. However, due to the high attenuation of the ultrasonic signal along the acoustic path, the signal-to-noise ratio (SNR) is low and the zero-crossing technique may not work well under all circumstances. Furthermore, a fluctuating offset could shift the zero crossing point and cause unacceptable errors [21]. When the received ultrasonic signal is severely disturbed by the noise, or interfered by spurious signals, the zerocrossing technique can lead to incorrect results. This is also the case in many industrial installations or environments with difficult fluids, e.g., extreme temperatures or highly polluted liquids and gases (such as sewage). In those situations, the cross-correlation method becomes particularly useful. Despite the cross-correlation method requires a higher computational cost, it has the advantage of being a very robust scheme while suppressing the disturbance of the noise. To compute the TOF, the received signal needs to be correlated with a reference signal. The TOF is determined by the position of the maximum of the cross-correlation function. Therefore, the reference signal plays a key role in the measurement of TOF. Surprisingly, few literature have involved in discussing how to select an appropriate reference signal. The common solution is to take the received signal at static state as the reference wave. However, possible differences between the upstream and downstream received signals may lead to errors. An alternative "echo" method was provided by Brassier et al. [22] to determine the reference wave. Nonetheless, it is based on the capture of an echo and the signal is very weak. Recently, Zhou et al. [23] proposed an "average" method based on averaging multiple ultrasonic waves, that overcomes this limitations. However, after some time of operation, the flowmeter need to be re-calibrated and the reference wave may need to be updated, to ensure an optimal accuracy.

In this paper, a cross-correlation-based algorithm to compute the TOF with high precision is presented. A novel approach to determine the reference signal is proposed. Within this strategy, the analytical solution of an acoustically-forced underdamped oscillator model is used as a reference signal. The outcome of this procedure is compared with that obtained by using the "average" method. The determination of the volumetric flow rate using the proposed method was tested and the experimental results are analyzed and discussed.

\section{TIME-OF-FLIGHT ESTIMATION ALGORITHM}

An accurate calculation of TOF is an essential point in the flow rate measurement in UFM. Using the cross-correlation method, the TOF is given by the time $\tau$ at which $R_{X Y}(\tau)$ is maximum, where

$$
R_{X Y}(\tau)=\int_{-\infty}^{\infty} X(t) Y(t+\tau) d t
$$

is the cross-correlation function between two signals $X(t)$ and $Y(t) . X(t)$ is a reference signal and $Y(t)$ is the signal received by a transducer (either upstream or downstream). An appropriate reference wave must resemble the received signal as much as possible for optimal results. 
For discrete-time signals, the correlation function is implemented as

$$
R_{X Y}(m)= \begin{cases}\sum_{n=1}^{N-m} X(n+m) Y(n), & \text { for } m \geq 0, \\ \sum_{n=1}^{N+m} Y(n-m) X(n), & \text { for } m<0 .\end{cases}
$$

where $N$ is the number of elements in signals $X$ and $Y$, and $m$ is the index of elements in the cross-correlation function. The fast Fourier transform (FFT) permits easy implementation of the cross-correlation [8], provided that the number of signal samples is a power of 2 ,

$$
R_{X Y}(m)=\mathrm{FFT}^{-1}\left[\operatorname{FFT}[X(m)] \operatorname{FFT}[Y(m)]^{*}\right],
$$

where * stands for the conjugate. The TOF of an ultrasonic signal travelling from transducer $a$ to transducer $b$, is then computed by

$$
t_{a b}=t_{Y}(r-N+q) ;
$$

where $t_{Y}(i)$ denotes the time corresponding to index $i$ measured in signal $Y, r$ is the index of the position of the maximum of the cross-correlation, and $q$ is the index of the time at which the pulse is received, in the reference signal $X$. Consequently, the selection of a convenient reference signal is a key element in order to obtain precise results.

\section{A. Determination of the reference signal}

With the aim to determine the form of the reference signal, a simple model is proposed. The model assumes that piezoelectric actuators act as underdamped oscillators. In this way, the voltage generated at the receiver transducer (i.e. the reference signal) can be obtained analytically by computing the shape of the acoustic waves that reach the receiver. Firstly, an electric signal is applied to the transmitter transducer. As a result, the piezoelectric material in the transmitter vibrates, thereby generating damped oscillations in the form of acoustic waves. Those waves travel through the flowing medium, until they reach the target transducer. As a consequence, the target transducer is excited by the incoming acoustic waves and the mechanical vibrations are converted into a voltage by the piezoelectric effect. Therefore, the reference signal is defined as the solution of a forced oscillator, in which the external force is the acoustic wave received through the flowing medium. If the signal sent to the transmitter transducer is a rectangular pulse $U(t)$, with amplitude $A$ and width $t_{w}$, the form of the acoustic signal is given by the solution of the non-homogeneous differential equation

$$
\ddot{X}_{a s}+2 \xi \omega_{n} \dot{X}_{a s}+\omega_{n}^{2} X_{a s}=U(t),
$$

where the subscript as stands for "acoustic signal", $\omega_{n}=2 \pi f_{n}$ with $f_{n}$ the natural frequency of the piezoelectric material, $\xi$ is the damping coefficient and $U(t)$ is the electric signal sent to the transmitter transducer. $U(t)$ can be written as $U(t)=A\left[\Theta(t)-\Theta\left(t-t_{w}\right)\right]$, where $\Theta(t)$ is the Heaviside step function. A transient particular solution of (11) can be written as [24]

$$
X_{a s}(t)=A\left[g(t)-g\left(t-t_{w}\right)\right],
$$



Fig. 2. Amplitude of the electric signal received at the target transducer as a function of time. Pink line $\left(Y_{n}(t)\right)$ corresponds to the received signal obtained experimentally. Red line $(Y(t))$ corresponds to the received signal, filtered by (16) with $N_{\mathrm{MA}}=100$. Black line shows the prediction by the analytical solution (15). A comparison between the experimentally received signal and the prediction by the proposed model indicates a high degree of similarity between the two curves.

where

$$
g(t)=\left[1-e^{-a t}\left(\cos (b t)+\frac{a}{b} \sin (b t)\right)\right] \Theta(t),
$$

with $a=\xi \omega_{n}$ and $b=\omega_{n} \sqrt{1-\xi^{2}}$. Equation (12) represents the acoustic signal that travels through the flowing media, and is received by the target transducer. Since the transmitter and receiver transducers are identical, the form of the electric signal induced in the receiver transducer will be the solution of an underdamped oscillator (with the same physical parameters as the transmitter transducer), under an external force driven by the acoustic signal given by (12). That is,

$$
\ddot{X}+2 \xi \omega_{n} \dot{X}+\omega_{n}^{2} X=X_{a s}(t) .
$$

Therefore, the solution of (14) will resemble the electric signal provided by the receiver transducer, and will be designated as the reference signal. The analytical solution of (14) is detailed in Appendix $\mathrm{A}$ and is given by

$$
X(t)=\frac{A}{b}\left[T_{1}(t)-T_{2}(t)\right] \Theta(t) .
$$

In order to determine the validity of this approach, the solution (15) has been plotted and compared with the received electric signal. The presented model takes four independent parameters as input variables, namely $A$ (pulse amplitude), $t_{w}$ (pulse width), $f_{n}$ (transducer resonance frequency) and $\xi$ (damping coefficient). Numerical values of these parameters have been set according to the transducer and electric pulse characteristics. On one hand, the transmitter is excited with a rectangular pulse of $A=3.3 \mathrm{~V}$ and width $t_{w}=260 \mathrm{~ns}$. On the other hand, the received signal has been obtained experimentally using two equal transducers (one acting as transmitter and one acting as recevier) with a resonance frequency of $f_{n}=2.02 \mathrm{MHz}$ and a damping coefficient of $\xi=0.08$. The signal received experimentally presents low SNR, therefore a moving-average filter [25] have been implemented with the aim to visualize the form of the received 


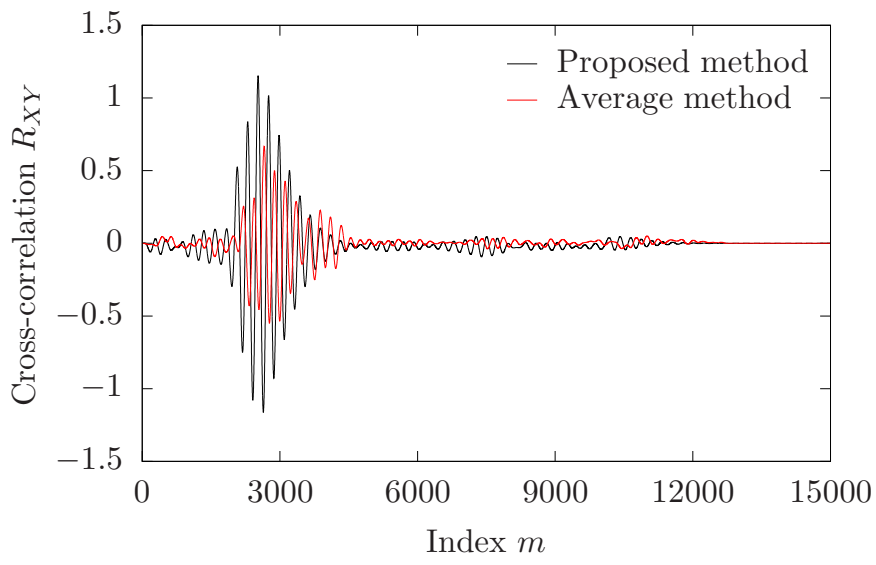

Fig. 3. Cross-correlation function using the average method and the analytical method. The position and the value of the maximum of the correlation function is different between the two methods.

signal without noise disturbances. The filtered signal at time $t_{i}$ is obtained by

$$
Y\left(t_{i}\right)=\frac{1}{N_{\mathrm{MA}}} \sum_{j=-N_{\mathrm{MA}} / 2}^{N_{\mathrm{MA}} / 2} Y_{n}\left(t_{i}+t_{j}\right),
$$

where $Y_{n}$ is the non-filtered signal received by the transducer, and $N_{\mathrm{MA}}$ is the number of points used to compute the moving average filter. The moving average filter has a significant advantage since it can be implemented with an algorithm that is very fast. First, there are only two computations per point, regardless of the length of the filter kernel. Second, addition and subtraction are the only math operations needed, while most digital filters require time-consuming multiplication. Third, the indexing scheme is very simple. Fourth, the entire algorithm can be carried out with integer representation. Depending on the hardware used, integers can be more than an order of magnitude faster than floating point. A comparison between the received signal $Y_{n}(t)$ obtained experimentally, the filtered signal $Y(t)$ using $N_{\mathrm{MA}}=100$, and the prediction by the proposed model given by (15), is presented in Fig. 2. Excellent agreement between the filtered signal and the theoretical predicition is achieved, despite the simplicity of the model.

\section{B. Comparison with the average method}

Within the average method, the reference wave is established by evaluating the average of ultrasonic signals acquired by both upstream and downstream transducers at zero flow conditions. Designating $Y_{u p}$ and $Y_{\text {down }}$ as the ultrasonic waves acquired by the upstream and downstream transducers respectively, the reference wave $Y_{\text {ave }}$ is computed by

$$
Y_{\text {ave }}=\frac{1}{2 N_{a}} \sum_{i=1}^{N_{a}} Y_{u p}^{i}+Y_{d o w n}^{i}
$$

where $N_{a}$ is the number of signals acquired.

The use of slightly different reference signals, each one correlated with the same received signal, results in considerably distinct forms of the corresponding cross-correlation functions.

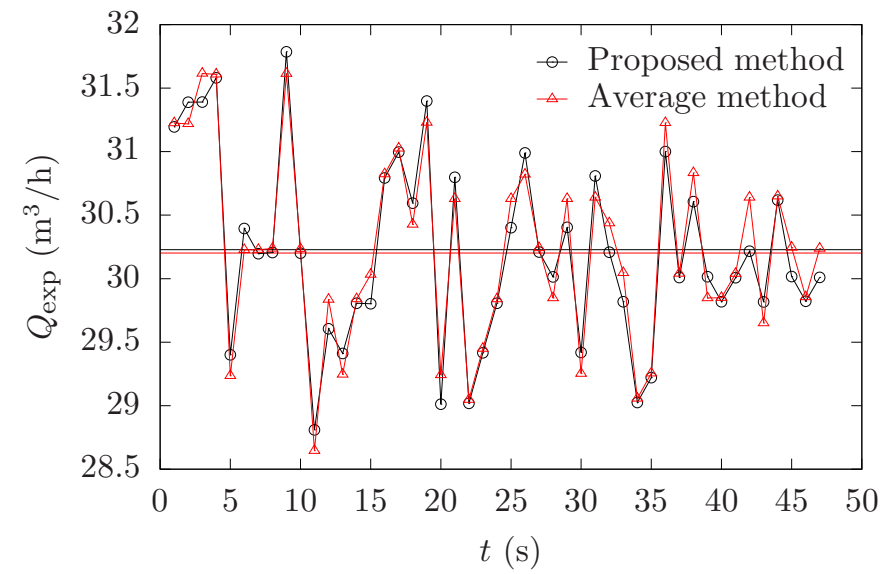

Fig. 4. Measured flow rate as a function of time. Red line corresponds to the flow rate computed using the average method, which uses an average curve obtained from multiple received waves, as the reference signal. The black line corresponds to the flow rate computed using the solution (15) as the reference signal. Horizontal lines correspond to the mean flow rate values.

A comparison between the cross-correlation functions, using the reference signal from (15) and using the reference signal obtained from the average method described in [23], is presented in Fig. 3. The average method have been used with an average of 12 signals: 4 of them obtained with $Q=0$ $\mathrm{m}^{3} / \mathrm{h}$ (static conditions), 4 with $Q=25 \mathrm{~m}^{3} / \mathrm{h}$ and 4 with $Q=50 \mathrm{~m}^{3} / \mathrm{h}$. The position of the maximum of the crosscorrelation is different using the two methods. Nonetheless, the value of the computed flow rate using the two methods is very similar, as can be observed in Fig. 4. The mean flow rate is $\left\langle Q_{p}\right\rangle=30.23 \pm 0.11$ for the proposed method, and $\left\langle Q_{a}\right\rangle=30.20 \pm 0.11$ for the average method. Here, the error has been computed as

$$
\varepsilon_{S D}=\sqrt{\frac{1}{N(N-1)} \sum_{i}\left(Q_{i}-\langle Q\rangle\right)^{2}} .
$$

Since the error associated to each case is larger than the difference between mean flow rate values, one can consider that the measured mean flow rates are equal for practical purposes, which indicates that the two approaches have similar accuracies.

The average method has proven to be very robust in a wide range of operational conditions. Nonetheless, it inherits a series of drawbacks such as: (i) the need of realizing multiple measurements for each acoustic path, previous to the designation of a proper reference wave; (ii) from time to time, the UFM needs to be re-calibrated and the reference wave may need to be updated. This increases substantially the maintenance costs of the UFM system; (iii) A reference wave should be obtained for each acoustic path, implying a set of large number of measurements for specific conditions. Hence, the average method lacks of portability and versatility. The model presented in this paper allows to determine an appropriate reference wave for a wide range of transducers: physical parameters such as the resonance frequency or the damping coefficient can be adjusted. Likewise, the form of the excitation pulse can be optimized according to the 
transducers used, without the need of multiple measurements. Consequently, the proposed procedure to determine the form of the reference wave, overcomes the main weaknesses of the average method while maintaining high accuracy.

\section{FLOW RATE MEASUREMENT}

The scheme to obtain reliable values of the flow rate is based on the following strategy: first, the values of TOF are obtained for each transmitted signal. If the flowmeter has multiple acoustic paths, a value of the local velocity is computed for each path using (3), such that

$$
v_{p_{i}}=\frac{D}{\sin \left(2 \alpha_{p_{i}}\right)} \frac{\Delta t_{p_{i}}}{\Pi t_{p_{i}}}
$$

where the $p_{i}$ subscript stands for the $i^{\text {th }}$ path. It is assumed that the pipe diameter $D$ is constant in all acoustic paths, but the separation length between transducers may change, leading to different values of $\alpha$ for each path. The experimental flow rate $Q_{\exp }$ is obtained as an average of the flow rates computed for each path, with the reintroduction of the calibration factor from (6)

$$
Q_{\exp }=K\left\langle Q_{p}\right\rangle
$$

where

$$
\left\langle Q_{p}\right\rangle=\frac{1}{N_{p}} \sum_{i} Q_{p_{i}}
$$

and

$$
Q_{p_{i}}=\frac{\pi D^{2}}{4} v_{p_{i}}
$$

being $N_{p}$ the number of acoustic paths.

Measured flow rate values using (20) exhibit a considerable fluctuation error, specially for high flow rates. For this reason, an additional "variable memory" filter have been implemented. The filter is based on a weighted average of the current and previous measured values of the flow rate values $Q_{\text {exp }}$ obtained by (20). Consequently, the filtered values of the flow rate obtained at a certain time $t_{i}$ are computed as

$$
Q\left(t_{i}\right)=\frac{1}{N_{\mathrm{VM}}} \sum_{j=1}^{N_{\mathrm{VM}}} \omega_{j} Q_{\exp }\left(t_{i}-t_{j}\right),
$$

with the following weight normalization

$$
\frac{1}{N_{\mathrm{VM}}} \sum_{j=1}^{N_{\mathrm{VM}}} \omega_{j}=1
$$

$N_{\mathrm{VM}}$ is the total number of points used to compute the final value of the flow rate. Designating $\omega_{j}=1$ for any value of $j$, is equivalent to a convolution with the measured $Q_{\exp }$ values and a rectangular pulse. Choosing high values of $N_{\mathrm{VM}}$ correspond to a considerable reduction of the fluctuation error, with a noticeable temporal delay in the measurements. As a matter of fact, the application of this filter allows to increase the resolution arbitrarily, at the expense of having a temporal delay in the UFM response.

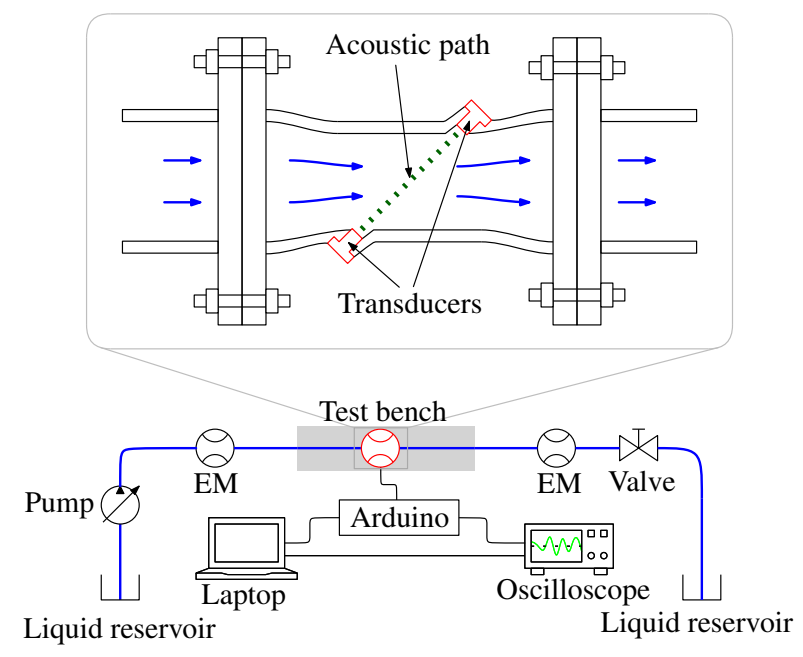

Fig. 5. Schematic diagram of the test pipe and sketch of the experimental setup. Pipe lengths before and after the test flowmeter are higher than ten times the pipe diameter. Two electromagnetic flowmeters are placed before and after the test flowmeter, to provide an accurate reference measurement.

\section{UFM EXPERIMENTAL RESULTS}

Experimental measurements were carried out using an UFM calibration test rig (Fig. 5). The proposed UFM system have been designed to be used in intrusive type flowmeters, and consists in 4 transducers ( 2 parallel acoustic paths forming an angle $\alpha=45^{\circ}$ with respect to the pipe axis) placed in a 100 $\mathrm{mm}$ nominal diameter ductile-iron body. The algortihm has been tested in four different flowmeter bodies (two DN100 and one DN50 commercial flowmeter bodies, and a DN100 custom prototype) with two sets of different transducers: a set of transducers used in commercial flowmeters with a resonance frequency of $4 \mathrm{MHz}$, and custom handmade transducers with a resonance frequency of $2 \mathrm{MHz}$. Results have been satisfactory in all the configurations tested. Pipe length before and after the test flowmeter are higher than ten times the pipe diameter, with a pipe thickness of $8.5 \mathrm{~mm}$. All the flowmeter bodies tested include a flow stabilizer at the inlet, except the custom prototype. Potable water at $19^{\circ} \mathrm{C}$ is pumped from a liquid reservoir into the test bench. The range of flow rates studied spans from $Q=0 \mathrm{~m}^{3} / \mathrm{h}$ to $Q=150 \mathrm{~m}^{3} / \mathrm{h}$. Two electromagnetic flowmeters are placed before and after the test flowmeter, in order to provide a reference value.

The active element in the handmade transducers consists of a piezoelectric material (PZT-based ceramics) in form of disc with $10 \mathrm{~mm}$ diameter and $1 \mathrm{~mm}$ thickness $\left(f_{n}=2.02 \mathrm{MHz}\right.$, thickness mode). Rectangular pulses ( $A=3.3 \mathrm{~V}, t_{w}=260 \mathrm{~ns}$ ) were sent to each transducer using an Arduino Due board. The time difference between pulses was programmed to be 200 $\mu s$. Therefore, the transducers doesn't work simultaneously: first, a pulse is sent to transducer 1 (and received a few $\mu$ s later in transducer 2). $200 \mu \mathrm{s}$ after sending the first pulse to transducer 1, a pulse is sent to transducer 2 . And the same for the other pair of transducers. The acoustic beam transmitted from one pair of transducers does not influence the other pair of transducers, since the acoustic beam generated by the transducers is highly collimated. Electric signals received 




Fig. 6. Measured flow rate as a function of time, in static conditions. Red line corresponds to the non-filtered values of the flow rate. Blue line corresponds to the filtered values of the flow rate, computed using the average method. Black line corresponds to the filtered values. The used filter in both cases is given by Eq. (23) with $N_{\mathrm{VM}}=25$ and $\omega_{j}=1$.

by each transducer were recorded using a Picoscope 6402C oscilloscope, with a sampling rate of $1.25 \mathrm{GHz}$.

In general, all flowmeters fail to measure accurately the flow rate at static conditions (near-zero flow). The absolute value of the relative errors increase drastically when the flow rate drops to near-zero values. This is an indicative that providing a wellcalibrated UFM in near-zero flows is relevant for the robustness of the measurement system. If the electrical parameters of the transducers are different (non-reciprocal conditions), a reference signal (with adjustable parameters) can be precomputed for each transducer if necessary. Then, the TOF can be measured by performing cross-correlations between the received signals and the reference signals. Nonetheless, even if the transucers are not identical in practice (specially with handmade transducers), the use of a single reference signal have been sufficient in all the tests performed, to obtain accurate values of the flow rate.

The dynamic range of modern flowmeters is defined as the ratio $R=Q_{3} / Q_{1}$, being $Q_{3}$ the nominal flow rate and $Q_{1}$ the minimum flow rate that can be measured with an error of $5 \%$ or less [26]. From a commercial point of view, it is of crucial importance to design a flowmeter system with high values of $R$, whereas keeping the production costs at reasonable limits. Therefore, the accurate measurement of low flow rates becomes a fundamental aspect for achieving a high dynamic range.

In Fig. 6, near-zero flow measurements using the proposed system are presented. The red line corresponds to the actual experimental flow rate computed by (20), whereas the solid black line corresponds to the filtered measurements obtained from (23) with $N_{\mathrm{VM}}=25$. The resolutions of the non-filtered and filtered measurements are $\Delta Q=0.076 \mathrm{~m}^{3} / \mathrm{h}$ and $\Delta Q=$ $0.003 \mathrm{~m}^{3} / \mathrm{h}$, respectively. Blue line in Fig. 6 corresponds to the filtered measurements obtained using the average method, and have been added for a visual comparison. As can be seen from Fig. 6, both reference waves computed by the analytical model and by the average method, lead to essentially equal

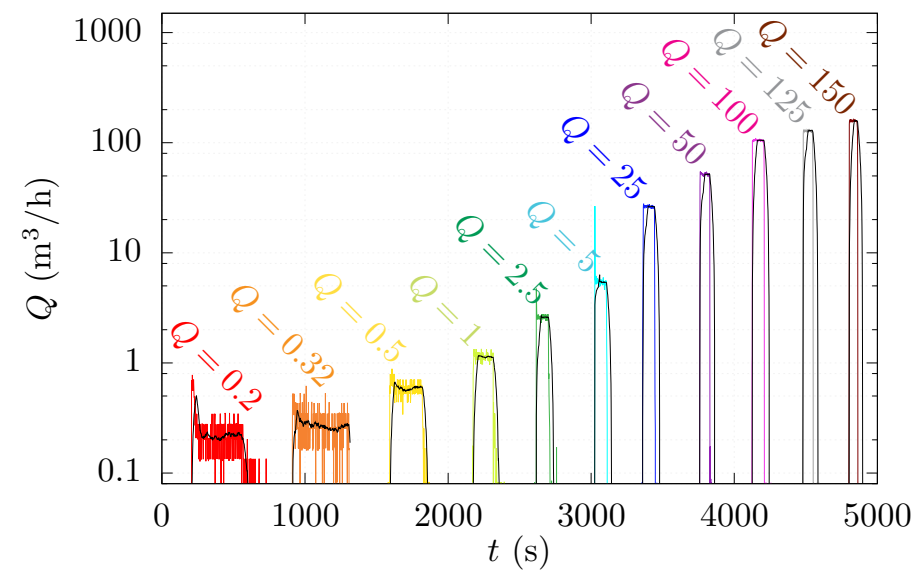

Fig. 7. Flow rate of the flow pulses as a function of time. Solid black line corresponds to filtered values, with $N_{\mathrm{VM}}=25$. Each flow pulse is used to obtain a value in the error curve of the proposed UFM. The flow pulse width decreases as the flow rate increases.

results.

The standard deviation of the TOF difference values have been computed under different flow rate conditions. Three values of the flow rate have been considered: $Q=1 \mathrm{~m}^{3} / \mathrm{h}$, $Q=10 \mathrm{~m}^{3} / \mathrm{h}$ and $Q=50 \mathrm{~m}^{3} / \mathrm{h}$. For each value of the flow rate, the time difference $\Delta t$ and its standard deviation $\sigma$ has been computed, using a total number of $N_{T}=752$ measurments (for the $Q=1 \mathrm{~m}^{3} / \mathrm{h}$ case), $N_{T}=638$ (for the $Q=10 \mathrm{~m}^{3} / \mathrm{h}$ case) and $N_{T}=420$ (for the $Q=50 \mathrm{~m}^{3} / \mathrm{h}$ case). The standard deviations obtained are $\sigma=0.98 \mathrm{~ns}$ (for the $Q=1 \mathrm{~m}^{3} / \mathrm{h}$ case), $\sigma=1.44 \mathrm{~ns}$ (for the $Q=10 \mathrm{~m}^{3} / \mathrm{h}$ case) and $\sigma=8.47 \mathrm{~ns}$ (for the $Q=50 \mathrm{~m}^{3} / \mathrm{h}$ case), hence as the flow rate increases, so does the standard deviation of the TOF difference.

To determine the precision of the used UFM, the error curve of the system must be obtained. In order to acquire the relative errors for each flow rate value, predefined-length pulses of flow are activated from static conditions. In this way, the total volume of flow is computed as

$$
V_{T}=\int_{0}^{W} Q(t) d t,
$$

where $W$ is the duration of the pulse of flow. In Fig. 7, a sample series of flow pulses used in the measurements are shown. Color lines correspond to the non-filtered experimental flow rate whereas the black line correspond to the filtered values, with $N_{\mathrm{VM}}=25$. It can be appreciated that there is a slight temporal delay in the response of the filtered values. However, this does not affect the total volume that flows in each pulse. The width of the flow pulse decreases as the flow rate increases, being of about $W \approx 10 \min$ for $Q=0.2 \mathrm{~m}^{3} / \mathrm{h}$ (resulting in a total volume of $V_{T}=32.2 \mathrm{~L}$ ) and $W \approx 1 \mathrm{~min}$ for $Q \approx 150 \mathrm{~m}^{3} / \mathrm{h}$ (resulting in $V_{T}=2518.2 \mathrm{~L}$ ).

Total volumes for each flow pulse were measured and compared to the real values, which were given by the calibration test rig facility. Results are presented in Fig. 8 in logarithmic scale. Very good agreement is achieved, since the measured points collapse at the curve $y=x$ (solid black line). This 


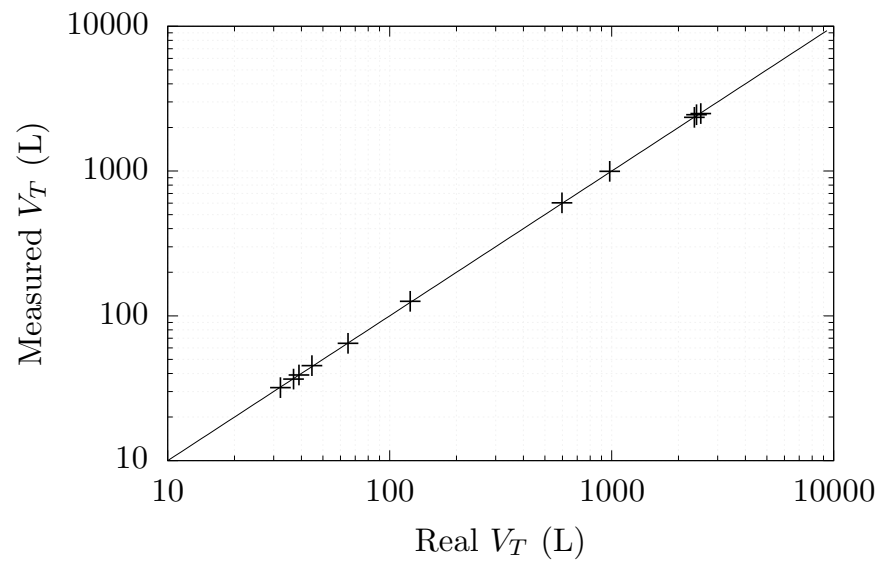

Fig. 8. Measured total volumes as a function of real total volumes (given by the calibration test rig facility). Black solid line corresponds to the curve $y=x$. Measured data lie above the $y=x$ curve, which indicates a high precision of the UFM system.

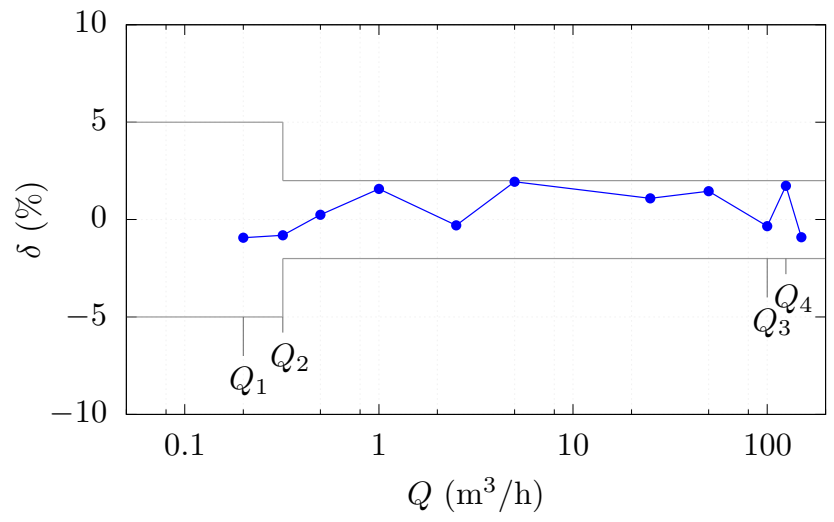

Fig. 9. Relative error as a function of the flow rate, for the proposed UFM system. The error curve lies below the $2 \%$ limit within all range of flow rates tested. Errors at low flow rates $\left(Q<1 \mathrm{~m}^{3} / \mathrm{h}\right)$ are noticeably low. Marked values $Q_{1}=0.2 \mathrm{~m}^{3} / \mathrm{h}, Q_{2}=0.32 \mathrm{~m}^{3} / \mathrm{h}, Q_{3}=100 \mathrm{~m}^{3} / \mathrm{h}$ and $Q_{4}=125$ $\mathrm{m}^{3} / \mathrm{h}$ have been plotted as reference values.

is a clear indication that the measurement system is highly accurate.

The error curve of the UFM system is presented in Fig. 9. The reference values $Q_{1}=0.2 \mathrm{~m}^{3} / \mathrm{h}, Q_{2}=0.32 \mathrm{~m}^{3} / \mathrm{h}$, $Q_{3}=100 \mathrm{~m}^{3} / \mathrm{h}$ and $Q_{4}=125 \mathrm{~m}^{3} / \mathrm{h}$ have been marked for comparison with the usual values of a flowmeter with the same nominal diameter and a dynamic range of $R=500$ [26]. Despite the fact that the error curve was obtained in one single test during a limited period of time, which could explain the instability at the high flow rate region, one can observe that the absolute value of all the relative errors are kept below $2 \%$. This reveals that the proposed system is highly precise, specially for low flow rates. Commonly, the relative error of general flowmeters increase remarkably at low values of the flow rate, reaching values higher than $5 \%$ for $Q<Q_{1}$. Hydraulic metrology industries are dedicating substantial efforts to gather UFM systems with high dynamic range $R>500$ while keeping low production costs. The algorithm proposed has proved to provide an encouraging value of $R>500$ (and can be even higher, if a wider range of flow rates are tested), which establishes the presented procedure as a promising candidate to be used in commercial UFM systems.

\section{CONCLUSION}

In this paper, an algorithm to determine the TOF using the cross-correlation technique is presented. A novel method to obtain an appropriate reference signal is also proposed. The reference signal is determined as the analytical solution of an acoustically-forced underdamped oscillator model, which consists in: (i) computing the form of the acoustic wave that travels through a flowing medium, considering the transmitter transducer as an underdamped oscillator; (ii) solving the underdamped oscillator equation, forced by the previouslycomputed acoustic wave; and, (iii) assuming that the signal received by the target transducer can be approximated by the solution of this forced underdamped oscillator equation, and designating its analytical solution as the reference signal. High similarity between the signal obtained experimentally and the prediction by the model is obtained. With the aim to validate the effectiveness of the method, an UFM system was tested with the proposed algorithm. A comparison with the average method revealed that both strategies provide similar accuracies. However, the proposed system has numerous advantages over the average method: (i) there is no need to realize multiple measurements for the determination of the reference wave; (ii) the maintenance of the UFM is unaffected: no re-calibration or additional measurements to update the reference wave are needed; (iii) the presented algorithm can be ported to many types of UFM systems that use a wide range of different transducers; (iv) reference wave characteristic parameters can be adjusted on-the-fly for optimal performance.

Measurements at static conditions have proven that the designed UFM system provides robust and reliable values at near-zero flows. This has the advantage of empowering the achievement of a high dynamic range $(R>500)$, with the potential to be even higher if a wider range of flow rates are explored. Flow rate measurement results prove that the algorithm can be used to determine the TOF with highprecision, in a wide range of flow rates. In particular, the absolute value of the relative errors of flow rate measurements were below $2 \%$ in all the range of flows considered (from 0.2 to $150 \mathrm{~m}^{3} / \mathrm{h}$ ). Further improvement can be achieved by using more acoustic paths on a cross-section of the pipe for velocity profile analysis.

\section{APPENDIX A}

ANALYTICAL SOLUTION OF THE ACOUSTICALLY-FORCED UNDERDMAPED OSCILLATOR MODEL

The electric signal provided by the receiver transducer is the solution of the non-homogeneous differential equation

$$
\ddot{X}+2 \xi \omega_{n} \dot{X}+\omega_{n}^{2} X=X_{a s}(t),
$$

where $X_{a s}(t)$ is the form of the acoustic signal travelling through the flowing medium,

$$
X_{a s}(t)=A\left[g(t)-g\left(t-t_{w}\right)\right],
$$


with

$$
g(t)=\left[1-e^{-a t}\left(\cos (b t)+\frac{a}{b} \sin (b t)\right)\right] \Theta(t),
$$

being $a=\xi \omega_{n}$ and $b=\omega_{n} \sqrt{1-\xi^{2}}$.

Fortunately, an analytical solution of (26) exists and can be obtained with the aid of the Green function,

$X(t)=\frac{A}{b} \int_{-\infty}^{t}\left[g(\tau)-g\left(\tau-t_{w}\right)\right] e^{-a(t-\tau)} \sin [b(t-\tau)] d \tau$.

Expanding the integral in two parts,

$$
\begin{aligned}
X(t) & =\frac{A}{b} \underbrace{\int_{-\infty}^{t} g(\tau) e^{-a(t-\tau)} \sin [b(t-\tau)] d \tau}_{:=T_{1}(t)}- \\
& -\frac{A}{b} \underbrace{\int_{-\infty}^{t} g\left(\tau-t_{w}\right) e^{-a(t-\tau)} \sin [b(t-\tau)] d \tau}_{:=T_{2}(t)} .
\end{aligned}
$$

The first term on the right-hand side of equation (30) is designated as $T_{1}(t)$, whereas the second term is denoted by $T_{2}(t)$. The Heaviside step function contained in $g(t)$ transforms the integration limits from $(-\infty, t]$ to $[0, t]$. Therefore, the term $T_{1}(t)$ can be rewritten as

$$
\begin{aligned}
T_{1}(t)=\int_{0}^{t}\left[1-e^{-a \tau}(\right. & \left.\left.\cos (b \tau)+\frac{a}{b} \sin (b \tau)\right)\right] . \\
\cdot & e^{-a(t-\tau)} \sin [b(t-\tau)] d \tau .
\end{aligned}
$$

Expanding the three terms in the parenthesis,

$$
\begin{aligned}
T_{1}(t)= & \int_{0}^{t} e^{-a(t-\tau)} \sin [b(t-\tau)] d \tau- \\
& -e^{-a t} \int_{0}^{t} \cos (b \tau) \sin [b(t-\tau)] d \tau- \\
& \quad-\frac{a e^{-a t}}{b} \int_{0}^{t} \sin (b \tau) \sin [b(t-\tau)] d \tau
\end{aligned}
$$

Integrating the three terms separately and rearranging, one obtains

$$
\begin{aligned}
T_{1}(t) & =e^{-a t}\left[\frac{b e^{a t}-a \sin (b t)-b \cos (b t)}{a^{2}+b^{2}}\right]- \\
& -e^{-a t}\left[\frac{t \sin (b t)}{2}-\frac{a[\sin (b t)-b t \cos (b t)]}{2 b^{2}}\right] .
\end{aligned}
$$

Following a similar procedure for the term $T_{2}(t)$, one arrives at

$$
\begin{gathered}
T_{2}(t)=\frac{e^{-a t}\left[b e^{a t}-a \sin (b t)-b \cos (b t)\right]}{a^{2}+b^{2}}- \\
-e^{-a\left(t-t_{w}\right)}\left[\frac{2 b t \sin \left[b\left(t-t_{w}\right)\right]+\cos \left[b\left(t_{w}-t\right)\right]-\cos \left[b\left(t_{w}+t\right)\right]}{4 b}\right. \\
-\frac{a e^{-a\left(t-t_{w}\right)}\left[\cos \left(b t_{w}\right) \sin (b t)-b t \cos \left[b\left(t-t_{w}\right)\right]\right]}{2 b^{2}}
\end{gathered}
$$

Rearranging terms, one can write the final form of the solution to (26), which is given by

$$
X(t)=\frac{A}{b}\left[T_{1}(t)-T_{2}(t)\right] \Theta(t) .
$$

\section{ACKNOWLEDGMENT}

The authors would like to acknowledge support from MINECO (Spanish Government) project ESP2015-72277EXP.

\section{REFERENCES}

[1] L. C. Lynnworth, and Y. Liu, "Ultrasonic flowmeters: half-century progress report, 1955-2005," Ultrasonics, vol. 44, pp. 1371-1378, 2006.

[2] P. Laugier and G. Haiat, "Bone Quantitative Ultrasound," Springer, 2011.

[3] G. Beldi, A. Bosshard, O. M. Hess, U. Althaus, and B. H. Walpoth, "Transit time flow measurement: experimental validation and comparison of three different systems," Ann. Thorac. Surg. vol. 70, pp. 212-219, 2000.

[4] H. Chiang, and C. L. Nikias, "Cumulant-based Adaptive Time Delay Estimation," Proc. of the 22nd Asilomar Conf. on Signals, Sys. and Comp., pp. 15-19, 1988.

[5] J. K. Tugnait, "Time delay estimation with unknown spatially correlated Gaussian noise," IEEE Trans. on Signal Proc., vol. 41, no. 2, pp. 549-558, 1993.

[6] M. Bezdek, H. Landes, A. Rieder and R. Lerch, "A coupled finite-element, boundary-integral method for simulating ultrasonic flowmeters," IEEE Trans. Ultrason., Ferroelectr., Freq. Control, vol. 15, pp. 10705-10722, 2015.

[7] W. Zhu, K. Xu, M. Fang, Z. Shen, and L. Tian, "Variable ratio threshold and zero-crossing detection based signal processing method for ultrasonic gas flow meter," Measurement, vol. 103, pp. 343-352, 2017.

[8] E. Mandard, D. Kouamé, R. Battault, J. P. Remenieras, and F. Patat, "Methodology for developing a highprecision ultrasound flow meter and fluid velocity profile reconstruction," IEEE Trans. on Ultrason., Ferroelectr., Freq. Control, vol. 55, pp. 161-172, 2008.

[9] Y. Chen, Y. Huang, X. Chen, "Acoustic propagation in viscous fluid with unifrom flow and novel design methodology for ultrasonic flow meter," Ultrasonics, vol. 53, pp. 595-606, 2013.

[10] C. Guoyu, G. Liu, B. Zhu, and W. Tan "3D isosceles triangular ultrasonic path of transit-time ultrasonic flowmeter: theoretical design and CFD simulations," IEEE Sensors J., vol. 15, no. 9, pp. 4733-4742, 2015.

[11] T. Petrut, C. Ioana, A. Digulescu, I. Candel, and G. Vasile "Flow rate computation of highly turbulent pipe flows using wide-band signals and matched filter-based approach in ultrasonic multi-element configuration," Proc. of the 10th Int. Conf. of Hydraulic Eff. Meas. IGHEM, pp. 12, 2014.

[12] G. Rajita, and N. Mandal "Review on transit time ultrasonic flowmeter," Proc. of the 2nd Int. Conf. on Control, Instr., Ener. \& Comm. (CIEC), Kolkata, pp. 88-92, 2016. 
[13] B. Mirshab, "Ultrasonic sensing for water flow meters and heat meters," Texas Instruments App. Rep., SNIA020, 2015.

[14] I. Murgan, C. Ioana, I. Candel, A. Anghel, J. L. Ballester, B., Reeb, B., and G. Combres, "(A new time of flight) Acoustic flow meter using wide band signals and adaptive beamforming techniques," IOP Conf. Series: Earth and Env. Science, vol. 49, 062002, 2016.

[15] K. Tawackolian, O. Buker, J. Hogendoorn, and T. Lederer "Calibration of an ultrasonic flow meter for hot water," Flow Meas. and Instr., vol. 30, pp. 166-173, 2013.

[16] Y. Bo, C. Li, L. Yupin, "Forced oscillation to reduce zero flow error and thermal drift for non-reciprocal operating liquid ultrasonic flowmeters," Flow Meas. and Instr., vol. 22, pp. 257-264, 2011.

[17] T. Folkestad and K. S. Mylvaganam, "Chirp excitation of ultrasonic probes and algorithm for filtering transit times in high-rangeability gas flow metering," IEEE Trans. on Ultrason., Ferroelectr., Freq. Control, vol. 40, no. 3 pp. 193-212, 1993.

[18] M. Takamoto, H. Ishikawa, K. Shimizu, H. Monji, and G. Matsui, "New measurement method for very low liquid flow rates using ultrasound," Flow Meas. and Instr., vol. 12, pp. 267-273, 2001.

[19] M. Bahram, "Ultrasonic sensing for water flow meters and heat meters," Texas Instruments Application Report, SNIA-020, Dallas, TX, USA, 2015.

[20] A. Hamouda, O. Manck, M.-L. Hafiane and N.-E. Bouguechal, "An enhanced technique for ultrasonic flow metering featuring very low jitter and offset," Sensors, vol. 16, pp. 1008-1020, 2016.

[21] A. B. Raine, N. Aslam, C. P. Underwood and S. Danaher, "Development of an ultrasonic airflow measurement device for ducted air," Sensors, vol. 15, pp. 10705-10722, 2015.

[22] P. Brassier, B. Hosten and F. Vulovic, "High-frequency transducers and correlation method to enhance ultrasonic gas flow metering," Flow Meas. and Instr., vol. 12, pp. 201-211, 2001.

[23] H. Zhou, T. Ji, R. Wang, X. Ge, X. Tang and S. Tang, "Multipath ultrasonic gas flow-meter based on multiple reference waves," Ultrasonics, vol. 82, pp. 145-152, 2018.

[24] B. A. de León and M. Mossberg, "Identification of under-damped second-order systems using finite duration rectangular pulse inputs," Proc. of the 2007 Am. Control Conf. 1-4244-0989-6, 2007.

[25] S. W. Smith, “The Scientist and Engineer's Guide to Digital Signal Processing," Newnes, 2002.

[26] Water meters for cold potable water and hot water. Metrological and technical requirements. ISO Standard 4064-1:2017. 